\title{
Risk factors related to oral candidiasis in patients with primary Sjögren's syndrome
}

\author{
Julia Serrano ${ }^{1,2}$, Rosa María López-Pintor ${ }^{1}$, Lucía Ramírez ${ }^{1}$, Mónica Fernández-Castro ${ }^{3}$, Mariano Sanz ${ }^{1}$, \\ Sheila Melchor ${ }^{4}$, Diana Peiteado ${ }^{5}$, Gonzalo Hernández ${ }^{1}$
}

\footnotetext{
${ }^{1}$ Department of Dental Clinical Specialties, ORALMED research group, School of Dentistry, Complutense University, Madrid, Spain

${ }^{2} \mathrm{PhD}$ Student, Research training fellow, Complutense University, Madrid

${ }^{3}$ Rheumatology Service, Hospital Puerta de Hierro, Madrid, Spain

${ }^{4}$ Rheumatology Service, Hospital Doce de Octubre, Madrid, Spain

${ }^{5}$ Rheumatology Service, Hospital La Paz, Madrid, Spain
}

Correspondence:

Departamento de Especialidades Clínicas Odontológicas

Facultad de Odontología, Universidad Complutense de Madrid

Plaza Ramón y Cajal s/n, 28040 Madrid, Spain

rmlopezp@ucm.es

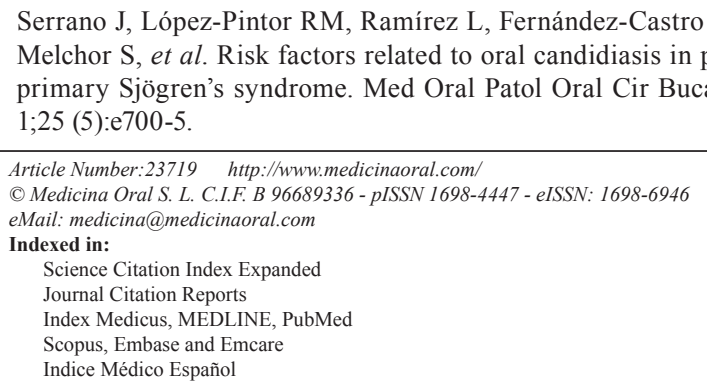

\begin{abstract}
Background: Candidiasis is the most frequent mycotic infection of the oral cavity. The aim of this study was to investigate the presence of clinical oral candidiasis and Candida albicans yeast in a population diagnosed of primary Sjögren's syndrome (pSS) and to study the possible factors associated with this infection. Material and Methods: An observational cross-sectional study was conducted in 61 pSS patients (60 women, 1 man, mean age 57.64 \pm 13.52 ) where patient based information (demographic and medical, tobacco and alcohol consumption history), intraoral parameters (presence of dentures, clinical signs of candidiasis), salivary analytical information (number of Candida albicans as colony-forming units per millilitre $(\mathrm{CFU} / \mathrm{mL}$ ), salivary $\mathrm{pH}$ levels, unstimulated whole saliva (UWS) and stimulated whole saliva (SWS) were collected. Results: $13.1 \%$ of pSS patients presented oral signs of candidiasis. Denture stomatitis and angular cheilitis were the most common lesions. $87.5 \%$ of patients with clinical candidiasis presented reduced $\mathrm{pH}$ levels and salivary flow in both UWS and SWS. A significant statistical negative correlation was found between CFU $/ \mathrm{mL}$ of Candida albicans and levels of UWS and SWS. A negative correlation was found between $\mathrm{pH}$ levels and $\mathrm{CFU} / \mathrm{mL}$, although not statistically significant. Conclusions: A reduced salivary flow may predispose pSS patients to Candida albicans overgrowth, which may show with clinical signs. Preventive measures are of great importance to avoid and to treat this condition promptly.
\end{abstract}

Key words: Sjögren's syndrome, oral candidiasis, oral lesions, Candida albicans, oral yeast, salivary flow rate, hyposalivation. 


\section{Introduction}

Primary Sjögren's syndrome (pSS) is a systemic autoimmune exocrinopathy that damages the salivary and lacrimal glands, resulting in dry eyes and hyposalivation $(1,2)$. Saliva contains IgA, lysozyme and lactoferrin, which are important antimicrobial defence mechanisms. Moreover, proper levels of saliva allow the lubrication of the mucosa and its buffering capacity maintains a physiological $\mathrm{pH}$ within the oral cavity $(3,4)$. In pSS patient's salivary gland hypofunction reduces the concentrations of immunoglobulins and other electrolytes (5), thus making the mucous membranes more exposed to the oral microbiota, and specifically to Candida infections (6). Candida species are commensal yeast present in the oral flora of healthy population. Nevertheless, in SS patients its prevalence has been estimated to be higher $(7,8)$. Therefore, simple identification of Candida yeast does not prove any infection and it is not always associated with the presence of clinical oral candidiasis (9). Candidiasis is the most frequent mycotic infection of the oral cavity, and it is usually diagnosed clinically, based on recognition of related lesions (9). The pathogenesis of this infection is still not fully understood, but a variety of systemic (as immunosuppression or endocrine disorders) and local factors (reduced salivary flow, use of dentures, high sugar diet, among others) have been associated to an overgrowth of Candida species, being Candida albicans the species most often associated with oral lesions $(10,11)$. This variety of predisposing factors alters to an environment that favours proliferation of Candida and leads to its transition from commensal to pathogenic, which may show with clinical signs and symptoms of oral candidiasis (9). The reported prevalence of clinical oral candidiasis in SS has varied widely $(0 \%-80 \%)$, mainly due to three factors: the lack of a clear symptomatology, patient related factors (such as oral hygiene habits) and different criteria used for diagnosing oral candidiasis in the literature (12).

Therefore, the main objective of this observational study was to investigate in a cohort of patients with pSS the association between the presence of $C$. albicans and clinical lesions of oral candidiasis with their salivary flow rates and $\mathrm{pH}$ levels. We also studied the possible influence of patient-related factors in the development of clinical oral candidiasis.

\section{Material and Methods}

- Study design, setting and subjects

A cross-sectional observational study was conducted following STROBE guidelines, as part of the EPOXSSp project (13). The patient cohort was the same as in a previous study carried out by this research group (14).
This sample consisted on consecutive patients who attended at different rheumatology services in the Community of Madrid (Spain) and which met the following inclusion criteria: being over 18 years old and being diagnosed of pSS according to the diagnostic criteria proposed by the American European Consensus Group (AECG) in 2002 (15). If selected patients had any other connective tissue disease or difficulties to attend to the School of Dentistry were excluded.

- Clinical variables and clinical diagnosis of oral candidiasis

A standard clinical protocol was applied and the following variables were recorded:

a) Patient related: age and gender, medical history, type and number of medicines, alcohol and tobacco consumption and wear and type of denture.

b) pSS disease: pSS AECG-2002 diagnostic criteria, time from diagnosis, serological data (rheumatoid factor, immunoglobulins alteration, antinuclear autoantibodies), and systemic manifestations of pSS (parotid enlargement, musculoskeletal, skin, lung, renal, central nervous system, peripheral nervous system, haematological, gastrointestinal or cardiac involvement).

c) Oral symptoms: xerostomia, dysphagia, dysgeusia and glossodynia.

A complete facial and intraoral mucosa examination was carried out by one calibrated specialist in oral medicine (JS). Diagnosis of clinical oral candidiasis was based on presence of the following clinical presentations: pseudomembranous candidiasis, acute erythematous candidiasis, chronic erythematous candidiasis (denture stomatitis), chronic hyperplastic candidiasis and secondary forms of oral candidiasis (angular cheilitis, median rhomboid glossitis and chronic mucocutaneous candidiasis) (11).

- Saliva Sampling

Stimulated (SWS) and unstimulated saliva (UWS) were collected by one specialist in oral medicine (LR). Patients were told not to eat, drink, smoke or brush their teeth 90 minutes prior the appointment. Samples were obtained in the morning, between 8.00 and $10.00 \mathrm{am}$, with the UWS sample always collected first during 15 minutes. To collect the SWS sample patients were asked to chew a paraffin gum and continuously spit out the saliva into a plastic container for 10 minutes. Flow rates were recorded as $\mathrm{mL} / \mathrm{min}$. Hyposalivation was defined as a flow rate $<0.7 \mathrm{~mL} / \mathrm{min}$ for SWS and $<0.1 \mathrm{~mL} / \mathrm{min}$ for UWS (14). To measure the salivary $\mathrm{pH}$ a $\mathrm{pH}$ meter was used (MicroPh2001, CRISON).

- Microbial sampling, culturing, and quantification of yeasts

Microbial samples were taken by rubbing a sterile cotton swab along the dorsal surface of the tongue of all patients. All swabs were taken by one operator (JS) before the saliva collection. Samples were transferred 
into a vial containing $1 \mathrm{~mL}$ RTF (reduced transport fluid), vortexed for 30 seconds in a mixer for homogenizing the collection and then aliquots of $0.02 \mathrm{~mL}$ of suspension were plated onto Sabouroud Agar plates (Sabouraud Dextrose Agar- DIFCO) and incubated at $37^{\circ} \mathrm{C}$ for $24-$ 48 hours. After incubation, colony-forming units per millilitre $(\mathrm{CFU} / \mathrm{mL})$ of $C$. albicans were counted. All samples were analysed at the Microbiology Laboratory of Faculty of Odontology at the University Complutense of Madrid.

\section{- Statistical analysis}

The sample size calculation (shown in a previous study by the same research group) (14) was calculated considering the data on prevalence of oral candidiasis (29.9\%) reported by Likar-Manookin et al. (3). Since this study did not compare oral candidiasis with a control group we used the percentage of oral candidiasis of the study of López-Pintor et al., which also compared the presence of oral candidiasis in renal transplant patients with a control group (4.19\%) using the same methodology (16). If we consider an $\alpha=0.05$ and a statistical power of $90 \%, 35$ patients will be needed in each group. If we consider a 15\% loss, 41 patients will be needed per group.

The categorical variables were described by their number and percentage and the quantitative variables by their media \pm standard deviation (SD). The KolmogorovSmirnoff test was applied to establish their goodness of fit to normality. To determine the differences between those pSS patients suffering from clinical oral candidiasis from those not suffering in regards to the categorical variables, the chi-squared test or Fisher's exact test were used. When assessing the differences in the quantitative variables, the U Mann-Whitney test was used. Spearman's rank correlation (r) coefficients were calculated to explore the associations between the quantitative variables. Differences were considered significant if $\mathrm{p}$ was $\leq 0.05$. All data were analysed using SPSS 25.0 for Windows.

\section{Results}

- Participants and descriptive data

All demographic data, pSS characteristics, systemic and serological manifestations from the selected patients are depicted in Table 1.

- Oral candidiasis in pSS

Clinical signs of oral candidiasis were present in $13.1 \%$ of pSS patients. Denture stomatitis was the most prevalent lesion (8.2\%), followed by angular cheilitis (4.9\%) and median rhomboid glossitis (1.6\%). No relationship was found between the clinical signs of oral candidiasis and the pSS variables. No other associations were observed between the rest of variables and the presence of clinical oral candidiasis (Table 1).
- Oral candidiasis and salivary flow rates

Table 2 shows the association between the presence of clinical oral candidiasis and UWS and SWS flow rates as well as with hyposalivation. pSS patients with oral candidiasis suffered more UWS and SWS hyposalivation and had lower flow rates compared with those without clinical oral candidiasis. A statistically significant association between clinical oral candidiasis and SWS hyposalivation was found.

- CFU/mL, salivary flow rates and $\mathrm{pH}$ levels

A statistically significant negative correlation was found between the amounts of $C$. albicans in $\mathrm{CFU} / \mathrm{mL}$ and the salivary flow levels of SWS $(r=-0.317 ; p=0.013)$ and UWS ( $\mathrm{r}=-0.339 ; \mathrm{p}=0.008$ ) (Fig. 1). A negative correlation between $C$. albicans and salivary $\mathrm{pH}$ levels was also found ( $\mathrm{r}=-0.225 ; \mathrm{p}=0.08)$, but without reaching statistical significance (Fig. 1).

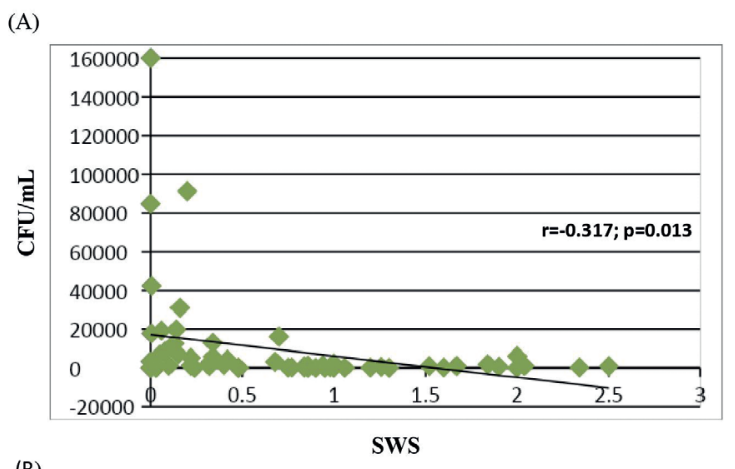

(B)

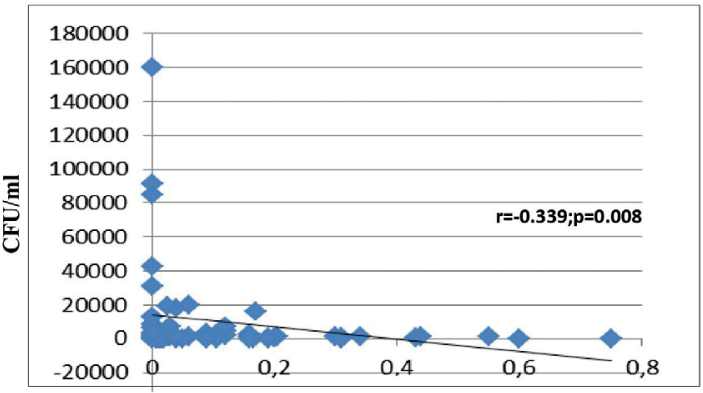

(C)

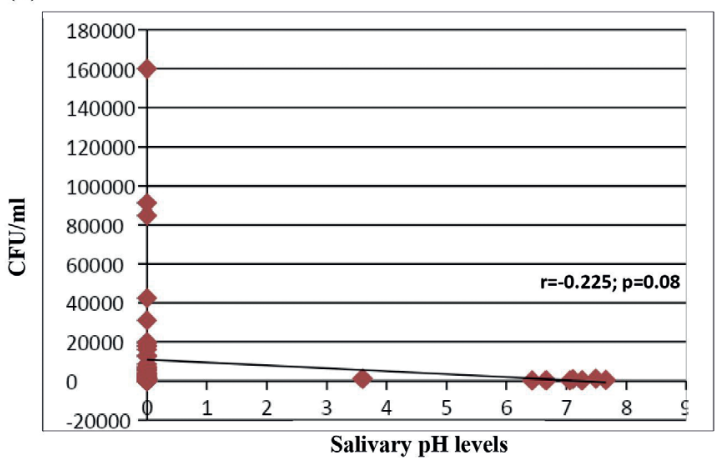

Fig. 1: (A) Correlation between SWS and Candida albicans CFU/ $\mathrm{mL}$, (B) Correlation between UWS and Candida albicans CFU/mL, (C) Correlation between salivary $\mathrm{pH}$ levels and Candida. 
Table 1: Differences between patients with and without oral candidiasis (OC)

\begin{tabular}{|c|c|c|c|}
\hline & Patients with OC $(n=8)$ & Patients without OC $(n=53)$ & $p$ \\
\hline \multicolumn{4}{|l|}{ pSS characteristics (AECG-2002) } \\
\hline Oral symptoms & $8(100 \%)$ & $51(96.2 \%)$ & $1^{\mathrm{c}}$ \\
\hline Ocular symptoms & $8(100 \%)$ & $53(100 \%)$ & $1^{\mathrm{c}}$ \\
\hline Oral signs & $5(62.5 \%)$ & $27(50.9 \%)$ & $0.71^{\mathrm{c}}$ \\
\hline Ocular signs & $7(87.5 \%)$ & $46(86.8 \%)$ & $0.95^{\mathrm{b}}$ \\
\hline Histopathology & $6(75 \%)$ & $26(49.1 \%)$ & $0.26^{\mathrm{c}}$ \\
\hline Autoantibodies (anti-Ro/ anti-La) & $7(87.5 \%)$ & $48(90.6 \%)$ & $0.78^{\mathrm{b}}$ \\
\hline \multicolumn{4}{|l|}{ pSS systemic manifestations } \\
\hline Whatever systemic manifestations & $5(62.5 \%)$ & $28(52.8 \%)$ & $0.7^{\mathrm{c}}$ \\
\hline Parotid enlargement & $4(50 \%)$ & $16(30.2 \%)$ & $0.26^{\mathrm{b}}$ \\
\hline Musculoskeletal involvement & $1(12.5 \%)$ & $28(52.8 \%)$ & $0.05^{\mathrm{c}}$ \\
\hline Skin involvement & 0 & $14(26.4 \%)$ & $0.09^{\mathrm{b}}$ \\
\hline Lung involvement & $1(12.5 \%)$ & $4(7.5 \%)$ & $0.51^{\mathrm{c}}$ \\
\hline Renal involvement & 0 & $3(5.7 \%)$ & $1^{\mathrm{c}}$ \\
\hline Central nervous system involvement & 0 & $1(1.9 \%)$ & $1^{\mathrm{c}}$ \\
\hline Peripheral nervous system involvement & 0 & $2(3.8 \%)$ & $1^{\mathrm{c}}$ \\
\hline Haematological involvement & $3(37.5 \%)$ & $15(28.3 \%)$ & $0.59^{\mathrm{b}}$ \\
\hline Gastrointestinal involvement & 0 & $3(5.7 \%)$ & $1^{\mathrm{c}}$ \\
\hline Cardiac involvement & 0 & 0 & - \\
\hline \multicolumn{4}{|l|}{ pSS serological manifestations } \\
\hline FR+ & $3(37.5 \%)$ & $30(56.6 \%)$ & $0.45^{\mathrm{b}}$ \\
\hline ANA+ & $7(87.5 \%)$ & $49(92.5 \%)$ & $0.5^{\mathrm{b}}$ \\
\hline Modified immunoglobulins & $5(62.5 \%)$ & $31(58.5 \%)$ & $1^{\mathrm{c}}$ \\
\hline \multicolumn{4}{|l|}{ Other variables } \\
\hline Smokers & 0 & $8(15.1 \%)$ & $0.58^{\mathrm{c}}$ \\
\hline Alcohol intake & $3(37.5 \%)$ & $10(18.9 \%)$ & $0.35^{\mathrm{c}}$ \\
\hline Antidepressant treatment & $1(12.5 \%)$ & $6(11.3 \%)$ & $0.92^{\mathrm{b}}$ \\
\hline Antihypertensive treatment & $1(12.5 \%)$ & $11(20.8 \%)$ & $1^{\mathrm{c}}$ \\
\hline Diabetes patients & 0 & $3(5.7 \%)$ & $1^{\mathrm{c}}$ \\
\hline Patients with dentures & $3(37.5 \%)$ & $10(18.9 \%)$ & $0.23^{\mathrm{b}}$ \\
\hline Acrylic denture & 0 & $5(9.4 \%)$ & $0.15^{\mathrm{b}}$ \\
\hline Metallic denture & $2(25 \%)$ & $3(5.7 \%)$ & $0.15^{\mathrm{b}}$ \\
\hline Implant-supported denture & $1(12.5 \%)$ & $1(1.9 \%)$ & $0.15^{\mathrm{b}}$ \\
\hline Acrylic and metallic denture & 0 & $1(1.9 \%)$ & $0.15^{\mathrm{b}}$ \\
\hline
\end{tabular}

Values are number (\%) of patients. OC: oral candidiasis; RF: Rheumatoid Factor; ANA: Antinuclear autoantibodies. Statistical test: ${ }^{\mathrm{b}} \mathrm{Chi}-\mathrm{squared}$ test or ${ }^{c}$ Fisher's exact test.

Table 2: Relationship between oral candidiasis and salivary variables.

\begin{tabular}{|c|c|c|c|}
\hline & pSS patients with OC $(n=8)$ & pSS patients without $\mathrm{OC}(\mathrm{n}=53)$ & $p$ \\
\hline \multicolumn{4}{|l|}{ UWS hyposalivation } \\
\hline Yes & $7(87.5 \%)$ & $30(56.6 \%)$ & \multirow{2}{*}{$0.09^{\mathrm{b}}$} \\
\hline No & $1(12.5 \%)$ & $23(43.4 \%)$ & \\
\hline UWS flow rate $\mathrm{mL} / \mathrm{min}($ mean $\pm \mathrm{SD})$ & $0.05 \pm 0.11$ & $0.13 \pm 0.17$ & $0.15^{\mathrm{a}}$ \\
\hline \multicolumn{4}{|l|}{ SWS hyposalivation } \\
\hline Yes & $7(87.5 \%)$ & $27(50.9 \%)$ & $0.05^{\mathrm{b}}$ \\
\hline No & $1(12.5 \%)$ & $26(49.1 \%)$ & \\
\hline SWS flow rate $\mathrm{mL} / \mathrm{min}($ mean \pm SD) & $0.36 \pm 0.69$ & $0.73 \pm 0.67$ & $0.08^{\mathrm{a}}$ \\
\hline
\end{tabular}

Values are number (\%) and mean \pm standard deviation (SD). Statistical test: ${ }^{\mathrm{a}} \mathrm{U}-\mathrm{Mann}-$ Whitney test, ${ }^{\mathrm{b}}$ Fisher's exact test. OC: oral candidiasis,

UWS: Unstimulated whole saliva, SWS: stimulated whole saliva. 
- Oral candidiasis and oral symptoms

$100 \%$ of pSS patients with clinical oral candidiasis also suffered xerostomia $(p=0.3), 50 \%$ dysphagia $(p=0.7)$ and glossodynia $(\mathrm{p}=0.27)$, but none referred dysgeusia $(p=0.28)$. The association between having clinical oral candidiasis and symptoms of oral discomfort was not statistically significant, although xerostomia was always present.

\section{Discussion}

This cross-sectional study on a cohort of pSS patients has found a significant inverse relationship between UWS and SWS levels and C. albicans counts. Hyposalivation is one of the main manifestations of SS. When salivary secretion is diminished, not only the volume of saliva decreases, but also its composition is altered, which may result in changes of the normal microflora homeostasis $(17,18)$. Hence, the predisposition of patients with decreased salivary flow to oral candidiasis could be attributed not only to the lack of mechanical washing activity but also to the lack of protective salivary factors (19-21). Hyposalivation has been associated with an increase in oral Candida counts (6), but, specifically in SS, it is not clear whether these high Candida levels are associated to lower UWS and/or decreased SWS flow rates. In fact, some authors have studied both types of saliva in pSS patients and have reported high Candida counts associated to both lower rates of SWS and UWS, although without demonstrating statistical significance $(22,23)$. Similarly, there are studies reporting that when C. albicans is higher UWS is decreased $(2,24,25)$, some demonstrating a significant relationship $(2,25)$. In other studies, however, the association was with SWS rather than UWS (26). In the present study, we have analysed both UWS and SWS and we have found a significant inverse relationship with $C$. albicans. These previous and present results emphasize the importance to frequently monitor the salivary flow rates in pSS patients to prevent superinfections by C. albicans. The clinical results from this study have further confirmed that hyposalivation is associated with clinical oral candidiasis. $87.5 \%$ of patients with clinical oral candidiasis presented SWS hyposalivation, compared to $50.9 \%$ pSS patients with a healthy mucosa. Similarly, $87.5 \%$ of patients with oral clinical candidiasis had UWS hyposalivation, compared to $56.6 \%$ without. As in previous reports (25) we have observed that pSS patients with low salivary flow rates had a higher risk for developing clinical oral candidiasis, with a statistical association between the SWS flow rate and oral candidiasis, however not with the UWS. In regard to other variables studied, we did not find any association between pSS related outcomes and clinical oral candidiasis. This is in agreement with Billings et al. who found that hyposalivation both UWS and SWS was significantly associated with clinical oral candidiasis, but not with other variables such as presence of autoantibodies and focus score (7).

It is accepted that Candida adherence to epithelial and acrylic surfaces is enhanced in the presence of low $\mathrm{pH}$ levels and salivary flow rate (27), nevertheless, very few studies have reported values of $\mathrm{pH}$ levels in SS patients (5). To our knowledge, this is the first report that compares $\mathrm{pH}$ levels in $\mathrm{pSS}$ patients with clinical signs of candidiasis or $C$. albicans loads. Low salivary $\mathrm{pH}$ values in SS may be caused by the low salivary flow rates and the decreased buffering capacity, although it is also possible that the progressive destruction of the salivary gland tissue may have a direct effect on the $\mathrm{pH}$ salivary secretion. In this investigation pSS patients with clinical oral candidiasis had lower $\mathrm{pH}$ levels $(4.40 \pm 2.91)$ than those without $(5.19 \pm 2.80)$ although these differences were not statistically significant. Similarly, when studying the association between $C$. albicans counts and $\mathrm{pH}$ levels, the lower the salivary $\mathrm{pH}$ the higher were the $C$. albicans counts. The lack of a significant association may be due to the variable ranges in $\mathrm{pH}$ described for a favourable Candida growth, between 3 to 8 (28). Another explanation may be due to the confounding effect of oral hygiene products, such as toothpastes or mouthwashes that compensate the drop in $\mathrm{pH}$ in these patients. More articles are needed to elucidate the possible relationship between $\mathrm{pH}$ levels and buffer capacity with Candida overgrowth and infection.

Although high Candida counts are predictive for clinical oral candidiasis (6), there is no relevant information on the critical yeast counts necessary to evidence the clinical signs of oral candidiasis (29). A recent study published by Zhou et al. (29) concluded that $266 \mathrm{CFU} /$ $\mathrm{mL}$ of $C$. albicans in saliva samples could be used as a threshold. In our study all patients with clinical signs of candidiasis had more than $266 \mathrm{CFU} / \mathrm{mL}$. New studies where the relationship of C. albicans counts and clinical signs of oral candidiasis should be carried out, in order to unify a cut-off point for considering C. albicans counts as an infection.

The present study has some limitations. The first is the cross-sectional nature of this investigation. Another limitation is the lack of a control non-pSS group. Besides, it should be noted that the low rate of clinical oral candidiasis observed in the current study may be due to good oral hygiene of the patients studied, $90 \%$ of included pSS patients brushed their teeth at least twice a day. In conclusion, this study shows how a lower salivary flow is related to higher counts of $C$. albicans. Since this oral condition may be symptomless, it is highly recommended the frequent monitoring and the emphasis of increased oral hygiene practices in these patients. Intervention prospective studies are needed to assess the impact of these enhanced oral preventive measures on the incidence of oral clinical candidiasis. 


\section{References}

1. Patel R, Shahane A. The epidemiology of Sjögren's syndrome. Clin Epidemiol. 2014;6:247-55.

2. Medeiros CCG, Borges LGDA, Cherubini K, Salum FG, Silva RMD, de Figueiredo MAZ. Oral yeast colonization in patients with primary and secondary Sjögren's syndrome. Oral Dis. 2018;24:1367-78. 3. Likar-Manookin K, Stewart C, Al-Hashimi I, Curtis W, Berg K, Cherian K, et al. Prevalence of oral lesions of autoimmune etiology in patients with primary Sjogren's syndrome. Oral Dis. 2013;6:598-603. 4. Shinozaki S, Moriyama M, Hayashida JN, Tanaka A, Maehara $\mathrm{T}$, Ieda $\mathrm{S}$, et al. Close association between oral Candida species and oral mucosal disorders in patients with xerostomia. Oral Dis. 2012;18:667-72.

5. Leung KC, McMillan AS, Leung WK, Wong MC, Lau CS, Mok TM. Oral health condition and saliva flow in southern Chinese with Sjögren's syndrome. Int Dent J. 2004;54:159-65.

6. Torres SR, Peixoto CB, Caldas DM, Silva EB, Akiti T, Nucci M, et al. Relationship between salivary flow rates and Candida counts in subjects with xerostomia. Oral Surg Oral Med Oral Pathol Oral Radiol Endod. 2002;93:149-154

7. Billings M, Dye BA, Iafolla T, Grisius M, Alevizos I. Elucidating the role of hyposalivation and autoimmunity in oral candidiasis. Oral Dis. 2017;23:387-94

8. Millsop JW, Fazel N. Oral candidiasis. Clin Dermatol. 2016;34:487-94. 9. Hertel M, Schmidt-Westhausen AM, Strietzel FP. Local, systemic, demographic, health-related factors influencing pathogenic yeast spectrum and antifungal drug administration frequency in oral candidiasis: a retrospective study. Clin Oral Investig. 2016;20:1477-86. 10. Oliveira MA, Carvalho LP, Gomes MdeS, Bacellar O, Barros TF, Carvalho EM. Microbiological and immunological features of oral candidiasis. Microbiol Immunol. 2007;51:713-9.

11. Williams D, Lewis M. Pathogenesis and treatment of oral candidosis. J Oral Microbiol. 2011;3:1-11.

12. Leung KC, McMillan AS, Cheung BP, Leung WK. Sjögren's syndrome sufferers have increased oral yeast levels despite regular dental care. Oral Dis. 2008;14:163-73.

13. Fernández-Castro M, López-Pintor RM, Serrano J, Ramírez L, Sanz M, Andreu JL, et al. Evaluación protocolizada odontológica en el paciente con síndrome de Sjögren primario. Proyecto EPOX-SSp: metodología y objetivos. Reumatol Clin. 2019.

14. Serrano J, López-Pintor RM, Fernández-Castro M, Ramírez, L, Sanz M, Casañas E, et al. Oral lesions in patients with primary Sjögren's syndrome. A case-control cross-sectional study. Med Oral Patol Oral Cir Bucal. 2020;25:e137-43.

15. Vitali C, Bombardieri S, Jonsson R, Moutsopoulos HM, Alexander EL, Carsons SE, et al. Classification criteria for Sjogren's syndrome: A revised version of the European criteria proposed by the American-European Consensus Group. Ann Rheum Dis. 2002;61:554-8

16. López-Pintor RM, Hernández G, de Arriba L, de Andrés A. Oral candidiasis in patients with renal transplants. Med Oral Patol Oral Cir Bucal. 2013;18:e381-7.

17. MacFarlane TW, Mason DK. Changes in the oral flora in Sjogren's syndrome. J Clin Path. 1974;27:416-19.

18. Shimizu C, Kuriyama T, Williams DW. Association of oral yeast carriage with specific host factors and altered mouth sensation. Oral Surg Oral Med Oral Pathol Oral Radiol. 2008;105:445-51.

19. Sweet SP, Denbury AN, Challacombe SJ. Salivary calprotectin levels are raised in patients with oral candidiasis or Sjögren's syndrome but decreased by HIV infection. Oral Microbiol Immunol. 2001;16:119-23.

20. Ergun S, Cekici A, Topcuoglu N, Migliari DA, Külekçi G, Tanyeri $\mathrm{H}$, et al. Oral status and Candida colonization in patients with Sjögren's Syndrome. Med Oral Patol Oral Cir Bucal. 2010;1:e310-5. 21. Serrano J, López-Pintor RM, González-Serrano J, FernandezCastro M, Casanas E, Hernandez G. Oral lesions in Sjögren's syndrome: a systematic review. Med Oral Patol Oral Cir Bucal. 2018;23:e391-400.
22. Lundström IM, Lindström FD. Subjective and clinical oral symptoms in patients with primary Sjögren's syndrome. Clin Exp Rheumatol. 1995;13:725-31.

23. Abraham MC, A1-Hashimi aI, Nasser Haghighat N. Evaluation of the levels of oral Candida in patients with Sjögren's syndrome. Oral Surg Oral Med Oral Pathol Oral Radiol. 1998;86:65-8.

24. Rhodus NL, Bloomquist C, Liljemark W, Bereuter J. Prevalence, density, and manifestations of oral Candida albicans in patients with Sjögren's syndrome. J Otolaryngol. 1997;26:300-6.

25. Yan Z, Young LA, Hua H, Xu Y. Multiple Oral Candida Infections in Patients with Sjögren's syndrome- Prevalence and Clinical and Drug Susceptibility Profiles. J Rheum. 2011;38:2428-31.

26. Radfar L, Shea Y, Fischer SH, Sankar V, Leakan RA, Baum BJ, et al. Fungal load and candidiasis in Sjögren's syndrome. Oral Surg Oral Med Oral Pathol Oral Radiol. 2003;96:283-7.

27. Kindelan SA, Yeoman CM, Douglas CWI, Franklin C. A comparison of intraoral Candida carriage in Sjögren's syndrome patients with healthy xerostomic controls. Oral Surg Oral Med Oral Pathol. 1998;85:162-7.

28. MacFarlane TW. The oral ecology of patients with severe Sjögren's syndrome. Microbios. 1984;41:99-106.

29. Zhou PR, Hua H, Liu XS. Quantity of Candida colonies in Saliva: A Diagnostic Evaluation for Oral Candidiasis. Chin J Dent Res. 2017;20:27-32.

\section{Acknowledgement}

We would like to thank the participation of the Investigators EPOXSSp Group: J.L Andreu (Hospital Puerta de Hierro Majadahonda), M.A Blázquez (Hospital Severo Ochoa), S. Recuero (Fundación Jiménez Díaz), J.A García (Hospital de la Princesa), G. Bonilla, L. Nuño (Hospital La Paz), T. Cobo, O. Illera, S. Muñoz, P. Richi, M. Steiner (Hospital Infanta Sofía), J.J González, M. Valero (Hospital San Chinarro), L. Lojo, M.T Navío (Hospital Infanta Leonor), F.J López (Hospital Gregorio Marañón), M. C Ortega (Hospital Infanta Elena), A Rodríguez (Hospital Ramón y Cajal), C Bohórquez (Hospital Príncipe de Asturias).

\section{Funding}

This research was supported in part by a grant from the Rheumatology Society of Comunidad de Madrid (SORCOM).

\section{Conflict of interest}

The authors report no conflict of interest.

\section{Ethics}

The clinical investigation was approved by the Ethical Committee of the University Hospital La Paz, Madrid (no: HULP PI-1891) with all procedures being carried out according to the Declaration of Helsinki and its following revisions.

\section{Authors contributions}

This study was designed by MFC and RMLP. The patients were recruited by the EPOX-SSp group rheumatologists. JS and LR carried out the oral examination of the patients. JS collected the data and RMLP analysed it. JS, RMLP, MS and GH wrote the paper. MFC, SM and DP reviewed and commented critically on the paper. All authors read and approved the final manuscript. 